\section{MUSCULOSKELETAL COMPLAINTS AMONG DENTAL PRACTITIONERS}

Cameron Estrich. American Dental Association, Chicago, Illinois, USA

\subsection{6/oemed-2014-102362.154}

Objectives To describe the prevalence and risk factors of musculoskeletal disorders among dental practitioners.

Method In 2012, 965 dental practitioners attending the American Dental Association Annual Session filled out surveys on individual characteristics and symptoms, then were clinically examined using the McKenzie evaluation method. Chi-square tests and linear regression were used to statistically compare associations between dental practitioner characteristics with musculoskeletal complaints.

Results $76.0 \%$ of practitioners regularly experienced pain, tingling, or numbness in their fingers, wrists, hands, forearms, shoulders, neck, back and chest during or at the end of a workday. Repetitive action during work was the most commonly reported origin of symptoms, reported in $37.9 \%$ of cases. $68.3 \%$ of practitioners reported that symptoms had prevented them from working. Symptoms located in the back were the most common, reported by $51.2 \%$. Location of symptoms was not related to gender, dominant hand, or age, but was related to specialty: dental hygienists and endodontists had significantly higher rates of back and hand symptoms than those in other dental fields (Chi-square p-values: 0.02, 0.002).

Years spent in practice was a significant predictor of the degree of symptoms (all F-test p-values $<0.05$ ). Equipment used during the workday affected complaints: magnifier use was associated with increased musculoskeletal symptoms, as were different operator chair types. Other significant predictors were exercising or stretching during the workday and self-reported working posture (Chi-square p-values: 0.002, 0.002).

Conclusions The dental profession experiences a high rate of musculoskeletal complaints. Ergonomic and educational interventions can prevent musculoskeletal complaints, and are clearly necessary for dental practitioners.

\section{SERUM HEME OXYGENASE-1 LEVEL IN SILICOSIS PATIENTS AND STONE MORTAR AND PESTLE PRODUCTION WORKERS}

${ }^{1}$ Kowit Nambunmee, ${ }^{2}$ Anongsin Danphaiboon, ${ }^{2}$ Jiaranai Khantipongse. ${ }^{1}$ Major of Occupational Health and Safety, School of Health Science, Mae Fah Luang University, Muang, Chiang Rai, Thailand; ' 2 The Office of Diseases Prevention and Control Region 10, Muang, Chiang Mai, Thailand

\subsection{6/oemed-2014-102362.155}

Objectives Silicosis is a chronic lung disease caused by silica dust exposure and is a significant health problem in industry involving stone crushing process such as mortar and pestle production industry. We proposed a use of Heme Oxygenase-1 (HO-1) to detect an early stage of silicosis. HO-1 is a lung inflammation and oxidative stress biomarker which has a potential to indicate silicosis. In this study, the serum level of HO-1 in exposed subjects (stone mortar and pestle production workers) was compared to a non-silica exposed subjects.

Method Total crystalline silica was quantified by ultraviolet visible spectrometry. A chest radiograph was performed in a General Hospital by a trained radiologist to detect silicosis according to International Labour Organisation guideline. The serum HO-1 level was determined by sandwich enzyme immunoassay.

Results There were 19 silicosis subjects in the exposed group which was categorised into the silicosis group. The process in stone mortar and pestle production industry produced high level of silica dust in the air ranging between $3.97-21.12 \mathrm{mg} / \mathrm{m}^{3}$. The level of HO-1 increased as the level of silica exposure increased even after adjusted for smoking and employment duration. The level of serum HO-1 level was, however, not significantly different between silicosis, exposed, and non-exposed groups, nor was the relationship between HO-1 and pulmonary function.

Conclusions We propose that HO- 1 reflects the lung inflammation caused by silica exposure although it is not specific to silicosis. HO-1 may be a useful parameter for monitoring lung inflammation in silica exposed population.

\section{OCCUPATIONAL EXPOSURE TO EXTREMELY LOW FREQUENCY MAGNETIC FIELDS OR ELECTRIC SHOCKS AND CANCER INCIDENCE IN FOUR NORDIC COUNTRIES}

${ }^{1}$ Monica Guxens, ${ }^{1}$ Pauline Slottje, ${ }^{1}$ Hans Kromhout, ${ }^{1}$ Anke Huss, ${ }^{2}$ Jan Ivar Martinsen, ${ }^{3}$ Timo Kauppinen, ${ }^{3}$ Sanni Uuksulainen, ${ }^{4}$ Elisabete Weiderspass, ${ }^{4}$ Pär Sparén, ${ }^{5}$ Laufey Tryggvadóttir, ${ }^{2}$ Kristina Kjærheim, ${ }^{1}$ Roel Vermeulen, ${ }^{6}$ Eero Pukkala. ${ }^{1}$ Institute for Risk Assessment Sciences, Utrecht, The Netherlands; ${ }^{2}$ Cancer Registry of Norway, Oslo, Norway; ${ }^{3}$ Finnish Institute of Occupational Health, Helsinki, Finland; ${ }^{4}$ Karolinska Institute, Stockholm, Sweden; ${ }^{5}$ /celandic Cancer Registry, Reykjavik, Iceland; ${ }^{6}$ Finnish Cancer Registry, Helsinki, Finland

\subsection{6/oemed-2014-102362.156}

Objectives To evaluate the association between occupational exposure to extremely low frequency magnetic fields (ELF-MF) or electric shocks and brain cancer, haemopoietic and lymphatic malignancies, and breast cancer incidence in the Nordic Occupational Cancer cohort.

Method The cohort was set up by linking occupational information from national censuses held in 1960, 1970, 1980-1, and/or 1990 to national cancer registry data in Finland, Iceland, Norway, and Sweden. All subjects aged 30-64 years who participated in a census were followed-up for cancer incidence until 2003-2005. Occupational exposure to ELF-MF or electric shocks (low, medium, high) was assigned to each subject based on census reported jobs using job-exposure matrices. For each case, five controls were randomly selected by matching for country, age, and sex. Conditional logistic regression models were performed adjusting for social class and occupational exposure to solvents.

Results A total of 68770 brain cancer cases, 65609 nonhodgkin lymphoma cases, 83088 leukaemia cases, 33791 multiple myeloma cases, 1827 male breast cancer cases, and 297,283 female breast cancer cases were included. Thirty-five percent of the total population was ever exposed to medium levels of ELF-MF and 7\% to high levels, whereas 19\% was ever exposed to a medium risk of electric shocks and $13 \%$ to a high risk. No associations were found between occupational exposure to ELF-MF or electric shocks and any of the cancer outcomes.

Conclusions In this very large census cohort we found no evidence of increased risk of several cancers in relation with occupational exposure to ELF-MF or electric shocks. 